\title{
O Processo do Conhecimento sobre a Gestão Estratégica de Pessoas no Contexto das Universidades
}

The Process of Knowledge in Strategic Human Resource Management in the Context of Universities

Luísa Karam de Mattos ${ }^{1}$, Luci Mari Aparecida Rodrigues², Anderson Digiácomo³ e Irineu Manoel de Souza ${ }^{4}$

1,2,3,4 Universidade Federal de Santa Catarina, UFSC 


\title{
Resumo
}

Esta pesquisa tem como objetivo caracterizar o processo do conhecimento sobre a gestão estratégica de pessoas no contexto das universidades. Para tanto, foi realizada uma revisão bibliográfica dos artigos publicados sobre a temática gestão estratégica e gestão de pessoas nas universidades, em periódicos indexados pelas bases Scopus ${ }^{\circledR}$ e Web of Science ${ }^{\circledR}$, no período de 2015 a 2020. Foram selecionados 13 artigos para a amostra, os quais apontam que tal campo de conhecimento apresenta potencial de pesquisa, bem como está em crescimento, devido ao aumento das publicações nos últimos anos de trabalhos sobre a temática, publicados em diversos países. Destaca-se a importância de estudos sobre o papel da liderança na estratégia relacionada a pessoas, bem como o interesse da academia pelo capital intelectual nas universidades. Por fim, ainda há que se ampliar discussões sobre o papel de outros agentes, para além dos gestores e docentes, bem como da lógica capitalista que atua coercitivamente sobre as universidades.

Palavras-chave: gestão estratégica, gestão estratégica de pessoas, gestão universitária

\begin{abstract}
This research aims to characterize the knowledge process that brings strategic management of people inside the university context. Considering this purpose, a bibliographic review of published articles on the topic of strategic management and people management in universities in high-impact international journals was performed, indexed by Scopus ${ }^{\circledR}$ and Web of Science ${ }^{\circledR}$, from 2015 to 2020. The research result brings 13 selected articles for the sample, which indicates that the field of knowledge has research potential, being as well in continuous growth, due to the increase in publications in recent years about these themes, published in several countries. The importance of studies on the role of leadership in strategy related to people is highlighted, as well as the interest of academia for intellectual capital in universities. Finally, it is still necessary to expand discussions on the role of other agents, in addition to managers and professors, as well as the capitalist logic that acts coercively on universities.
\end{abstract}

Keywords: strategic management, human resource management, university management 
Em uma sociedade onde o planejamento e a gestão eficiente dos diversos tipos de organizações e instituições se fazem tão necessários, determinados mecanismos são fundamentais para que isso aconteça de forma efetiva. Ao se pensar no longo prazo, a estratégia mostra-se fundamental para que as organizações venham a alcançar seus objetivos.

A gestão estratégica apresenta-se como um processo contínuo e adaptável, que contempla a compreensão das diferenças entre a estratégia realizada de uma organização com a estratégia pretendida, muito devido às mudanças nos diversos setores da sociedade (Herrero Filho, 2017; Mintzberg \& Waters, 1985). A partir disso, fazse necessário, ainda, o acompanhamento do que ocorre no ambiente em que tais organizações/instituições estão inseridas, observando-se tanto mudanças internas, quanto as que acontecem externamente, dado que ambas poderão gerar impactos no resultado estratégico esperado.

O foco deste estudo recai sobre o processo de gestão estratégica de pessoas dentro das instituições universitárias. Tais instituições possuem o Plano de Desenvolvimento Institucional, um planejamento de cinco anos que contempla pontos importantes na busca de seus objetivos e está pautado por estratégias inerentes às metas estipuladas. Considera-se, ainda, o horizonte de aperfeiçoamento e a ampliação das atividades que ocorrem dentro do ambiente universitário Lei n 10.861 (2004), o que, consequentemente, contempla a gestão estratégica de pessoas (Allui \& Sahni, 2016).

As universidades caracterizam-se como instituições vinculadas à geração de conhecimentos científicos, essenciais para orientar práticas relacionadas às profissões e aos demais tipos de organizações, bem como para a criação e adaptação de sistemas e métodos aplicáveis aos problemas advindos da sociedade, a fim de promoverem o desenvolvimento social, tecnológico e científico das nações.

Por meio de uma investigação acerca da realidade das universidades, busca-se ampliar as discussões sobre como ocorre a inserção da gestão estratégica em ambientes específicos, assunto que vem sendo explorado nas mais diversas áreas teórica e empiricamente (Jarzabkowski \& Kaplan, 2015; Juul Andersen \& Minbaeva, 2013; Mintzberg, Ahlstrand, \& Lampel, 1998). Mas, até o momento, sem uma análise direcionada à compreensão de como o processo de conhecimento voltado à realidade das universidades tem se desenvolvido, considerado o fato de que estas são ambientes dotados de características e complexidades peculiares e que contemplam perfis diversificados de pessoas (Souza, 2009).

Tal lacuna de investigação levou os autores ao seguinte questionamento: Como se caracteriza o processo do conhecimento sobre a gestão estratégica de pessoas no contexto das universidades? Assim, para responder ao proposto, objetiva-se caracterizar o processo do conhecimento sobre a gestão estratégica de pessoas no contexto das universidades.

Para atingir este objetivo, verificou-se o estado da arte internacional sobre gestão estratégica de pessoas nas universidades. Realizou-se uma busca por artigos publicados em periódicos indexados nas bases de dados Scopus $^{\circledast}$ e Web of Science ${ }^{\circledR}$, publicados no período de 2015 a 2020, e que continham as palavras-chave "strategic management", "strategic human resource management"; "higher education". 
Diante da relevância da problemática ora exposta, adotou-se a abordagem de Roesch (2009) em termos de dimensões para justificar este estudo, quais sejam: importância, oportunidade e viabilidade. Para a primeira, tem-se o fato de que a gestão estratégica tanto é considerada como uma área de estudo como de prática organizacional. Tal gestão abrange tanto objetivos estratégicos norteados por uma visão de processo (Mintzberg \& Waters, 1985) quanto de conteúdo em contextos de mudanças de natureza contingencial e que abarcam a ação das pessoas, o que a torna relevante para os diversos tipos de organizações (Mintzberg \& Lampel, 1999; Walker, 2013).

Embora haja um considerável corpo de estudos que priorize a análise de fenômenos organizacionais sob a lente teórica da gestão estratégica, com o enfoque nas empresas que atuam expressamente sob a lógica do desempenho voltado à competição de mercado, há que se reconhecer a importância desse tipo de gestão em outros tipos de organizações, tais como as universidades, que conforme assevera Jarzabkowski (2005), de fato são ambientes nos quais a gestão estratégica pode ser tratada, mas que devido a predominância da lógica de mercado, elas têm sido negligenciadas enquanto searas de fazer estratégico. Mas, esse tipo de instituição atende é ambiente fecundo para a temática. São, portanto, ambientes férteis para investigações, dada a complexidade que apresentam e a multiplicidade de objetivos e de agentes que nela estão inseridos (Easterby-Smith, 1987; Massen \& Potman, 1990; Rodrigues, Moreira, \& Martins, 2020).

Reforça-se o interesse deste estudo sob a oportunidade de apresentar reflexões em nível de processo de conhecimento sobre a inserção da gestão estratégica de pessoas nas universidades, destacando-se como os autores dos últimos cinco anos têm explorado as temáticas nelas presentes sob a lente teórica em comento. Ou seja, busca-se colaborar com o corpo de estudos que visam trazer à tona a realidade de instituições essenciais para o desenvolvimento dos países.

A premissa de estudo é a de que, diante as diversas pressões que as universidades enfrentam em seu ambiente de atuação, elas agem estrategicamente, o que envolve o sistema organizacional da gestão de pessoas no intuito de avançarem em seus objetivos, que contemplam tanto as áreas de ensino, pesquisa, extensão e gestão.

Desse modo, a viabilidade deste estudo está assegurada na disponibilidade de materiais e métodos que subsidiam a busca pela resposta ao objetivo proposto, sendo que as escolhas daqueles que serão aplicados constam detalhadas na seção de metodologia deste trabalho.

Por fim, esta pesquisa está estruturada por esta seção introdutória. Depois, são trazidos os referenciais teóricos que dão suporte à proposta de pesquisa, quais sejam: gestão estratégica, gestão estratégica de pessoas e a gestão estratégica de pessoas nas universidades. Em seguida, são expostos os procedimentos metodológicos escolhidos para a investigação. Na sequência, passa-se à apresentação e discussão dos resultados do estudo e, a partir deles expõe-se as considerações finais do trabalho e as referências utilizadas.

\section{Fundamentação Teórica}

\section{Gestão Estratégica}

Considerada a evolução da teoria da Estratégia, desde a suas origens na seara militar como arte para a conquista de batalhas (Kotler, Berger, \& Bickhoff, 2010) e sua posterior inserção no contexto organizacional, na 
década de 1930 (Barnard, 1938), denota-se a presença de múltiplos enfoques no processo de desenvolvimento dessa teoria, em que,

Na década de 1960, a Estratégia era equiparada ao planejamento corporativo, enquanto, nos anos de 1970, trabalhavam-se os aspectos inerentes à diversificação e ao planejamento de portfólio [...]. Por sua vez, da década de 1980 em diante concentrou-se no chamado negócio principal (core business), bem como, em visões menos analíticas e também voltadas às pessoas no contexto organizacional (Rodrigues, 2017, p. 46). Essas mudanças ocorreram e ainda acontecem em meio a contextos sociais, econômicos e tecnológicos diversos, que marcaram e seguem influenciando a visão de pesquisadores e de agentes que atuam estrategicamente, a respeito do que importa em termos expectativas de posição futura das organizações em cenários de mudanças, sejam elas contínuas ou não (Drucker, 1954; Golsorkhi, Rouleau, Seidl, \& Vaara, 2015; Mintzberg \& Lampel, 1999; Quinn, 1978).

A Estratégia tem se tornado uma teoria cada vez mais robusta e complexa, o que corrobora com a falta de consenso entre seus teóricos em relação a como ela deve ser conceituada (Rodrigues et al., 2020). Isso ocorre, exatamente, por suas inúmeras relações com a sociedade e a diversidade do pensamento humano, o que se estende aos pressupostos da Gestão Estratégica (Balogun, Jacobs, Jarzabkowski, Mantere, \& Vaara, 2014; Golsorkhi et al., 2015; Mintzberg \& Waters, 1985). Esta última contempla elementos estratégicos relacionados, exatamente, aos ambientes peculiares de interação humana nomeados como "organizações" (Etzioni, 1972).

Em seus aspectos constituintes, a Gestão Estratégica abrange um conjunto de elementos provenientes do pensamento estratégico e que são aplicáveis ao trabalho desempenhado no cotidiano das organizações, no intuito do atendimento de objetivos variados, os quais decorrem da busca por resultados que abrangem horizontes de curto, médio e longo prazo (Andersen, 2013; Ansoff, 1965; Chandler, 1962; Hamel \& Prahalad, 1994; Mintzberg et al., 1998; Porter, 1980).

Tais elementos são materializados por meio de três abordagens teóricas distintas da estratégia. A primeira, de conteúdo estratégico, vincula-se a perspectivas prescritivas e sistemáticas, voltadas à forma pela qual a estrutura (rígida) molda a estratégia, por meio da concepção e da tomada de decisão deliberada pela alta gestão das organizações (Chandler, 1962; Mintzberg \& Quinn, 2001; Porter, 1980). São utilizados mecanismos de controle e de desempenho, por meio de ferramentas como: a análise SWOT, as Core Competences, o Balanced Scorecard, as aplicações da Matriz BCG, a análise das Cinco Forças de Porter, o Planejamento Estratégico, planejamento de cenários, Strategic Dashboard, entre outras (Mintzberg et al., 1998; Qehaja, Kutllovci, \& Pula, 2017).

A segunda, de processo estratégico, além de conter aspectos prescritivos para a gestão estratégica, adota a natureza descritiva da estratégia. Ou seja, passa-se a abordar uma visão adaptativa, em que os processos de formação e de implementação da estratégia são vistos a partir do ambiente no qual as organizações se inserem. E, com isso, observa-se a influência do impacto de estratégias emergentes no fazer cotidiano, sob a égide da adequação ao contexto e ao cenário estratégico e da participação de múltiplos agentes no trabalho estratégico (Elbanna, 2006; Farjoun, 2002; Mintzberg \& Waters, 1985b; Qehaja et al., 2017). 
Por fim, a terceira perspectiva, caracteriza-se como integradora e não separa conteúdo de processo estratégico. Pois, ressalta a importância da complementaridade de ambas e, ainda, salienta que quando utilizadas separadamente, tais perspectivas tornam a gestão estratégica limitada, com prejuízos ao fazer cotidiano e de horizontes de planejamento nas organizações. Pois, dado que há uma influência mútua entre elas, sua utilização conjunta traz ganhos no processo de formação e de implementação estratégica (Elbanna, 2006; Huff \& Reger, 1987; Mintzberg \& Waters, 1985b; Pettigrew, 1992; Qehaja et al., 2017; Robinson Jr. \& Pearce II, 1988). A seguir, passa-se a tratar da gestão estratégica em sistemas organizacionais específicos e, a partir do interesse deste estudo, abordarse-á a gestão estratégica de pessoas.

\section{Gestão Estratégica de Pessoas}

Dentro deste tópico, cabe conceituar que, a Gestão de Pessoas (GP), segundo (W. J. A. Pedro, 2005, p. 83) “deve ocupar-se também da determinação de objetivos, de políticas, de diretrizes e da estruturação das organizações". O olhar sob essa área organizacional deve vir associado a pontos definidos anteriormente, para que eles sejam integrados aos processos de gestão, os quais passam a ser pautados por uma visão estratégica.

Para compreendermos o contexto de Gestão de Pessoas, no século XXI, devemos observar que o gestor atual deve ter atenção a alguns aspectos, como: econômico, social, político, jurídico, ambiental e cultural, além desses, os internos à organização, como logística interna, perfil dos colaboradores e a possibilidade de desenvolvimento de carreira, assim como o clima e a cultura organizacional (Boas \& Andrade, 2009).

Nessa direção, a Gestão Estratégica passou a contemplar alguns aspectos que considerassem o ambiente de Gestão de Pessoas. A partir desse movimento, surgiu a Gestão Estratégica de Pessoas, nos anos 1980, sob argumentações pautadas em considerações ao papel funcional, burocrático e fraquezas percebidas na área, por pressões do ambiente que apontavam para a natureza estratégica da área de Gestão de Pessoas e, também, dentro do processo de planejamento estratégico, que começou a levar em conta a área, buscando alcançar objetivos organizacionais (Dutra, 2014). É nesse contexto que a Gestão Estratégica de Pessoas se alinha aos objetivos da organização, preocupando-se não apenas com o setor de pessoal, mas, com todos os impactos que as decisões desse setor trarão a organização.

Há, dentro da área de Gestão Estratégica de Pessoas, alguns mecanismos e instrumentos principais, que entre eles estão o Planejamento de Recursos Humanos, a Gestão de Competências, a Capacitação Continuada com base em competências e a Avaliação de Desempenho e de Competências (Pantoja, Camões, \& Bergue, 2010). Estes têm por finalidade a parametrização do desempenho organizacional da área e o desenvolvimento das pessoas, consideradas as suas potencialidades e os objetivos a serem atingidos.

Em termos de organizações, o ambiente público, foco desta pesquisa, tem suas particularidades, assim como o privado, que os diferenciam. Dentro do ambiente público, embora reformas do estado tenham sido empreendidas, em geral, nesse setor as estratégias de Gestão de Pessoas são estabelecidas por métodos mais centralizados, cabendo às organizações se ajustarem internamente para o ambiente que estão, o que pode obstar a 
forma estratégica de agir, inclusive, sua própria autonomia, visto que precisam implementar estratégias e modelos de gestão definidos pelo Estado (da Fonseca, Meneses, Filho, \& Campos, 2013).

Vale ressaltar, ainda, que ferramentas são utilizadas para uma análise mais aprofundada da Gestão Estratégica de Pessoas em ambos os tipos de setor, dentre elas, o BSC; a sua aplicação permite que haja alinhamento entre as equipes de gestão, unidades de negócios, recursos humanos, tecnologia da informação e os recursos financeiros, de acordo com o que se busca através das estratégias organizacionais (Peris-Ortiz, GarcíaHurtado, \& Devece, 2019).

Estudos anteriores demonstram resultados importantes no uso de ferramentas estratégicas aplicadas à gestão de pessoas, como em Universidades da América Latina, em que houve significativo avanço no desempenho institucional, considerados indicadores de avaliação, promoção e remuneração. Estes proporcionam transparência processual e permitem aos gestores e colaboradores terem clareza no seu alinhamento com a instituição em que estão desenvolvendo seu trabalho (Peris-Ortiz et al., 2019).

Nas organizações modernas, que têm sua base na relação com o conhecimento, o planejamento estratégico, enquanto ferramenta, é um planejamento estratégico de recursos humanos. Há nele três elementos capazes de cobrir o sistema de Gestão de Pessoas, quais sejam: a) gestão: por meio de cargos e competências bem definidos; b) pessoal: a partir de planos de carreira e segurança quanto ao emprego; c) compensação: que traz questões baseadas em desempenho e eficiência (Daley, 2016). Portanto, observa-se que a gestão estratégica atual deve levar em conta os aspectos da gestão estratégica de pessoas para que seja completa e tenha mais chances de ser bem sucedida.

A seguir, passa-se a tratar da gestão estratégica de pessoas no ambiente das universidades.

\section{Gestão Estratégica de Pessoas nas Universidades}

O capital humano é considerado como uma vantagem sustentável nas organizações, considerado o mundo globalizado e em constante mudança (Bontis, 1996). Um estudo de Hasani e Sheikhesmaeili (2016) conclui que, mais do que nunca, por estarmos na era do conhecimento, o capital humano é mais importante do que as novas tecnologias ou recursos financeiros e materiais.

De acordo com Keller (1983), o ensino superior americano na década de 1980 entrou em uma nova era que exigia novos procedimentos e novas atitudes: melhor planejamento, tomada de decisões estratégicas e mudanças mais direcionadas na gestão de recursos humanos. Embora a universidade seja composta por servidores administrativos, professores e estudantes, quando se estuda a gestão de recursos humanos nas instituições de educação, a maioria das pesquisas estão focadas nos professores e em sua satisfação, ou seja, estratégias para reter o corpo docente nas instituições de ensino (Zhao \& Tao, 2017).

Alguns exemplos de estratégias na gestão de pessoas voltadas ao corpo docente são citadas por Zhao e Tao (2017): otimização da alocação de recursos humanos, formação de equipes acadêmicas de nível mundial para atrair os melhores talentos, realização de cooperação substantiva entre universidades e instituições de pesquisa 
famosas nacionais e estrangeiras, expandir as fontes de investimento em ensino superior, e carreiras vantajosas para professores em tempo integral.

A abordagem da gestão estratégica de pessoas nas universidades é tema de diversas pesquisas, porém o foco é o servidor docente. Entretanto, Bradley (2016) afirma que na gestão de pessoas é fundamental que os conceitos de gestão de talentos sejam aplicados em todos os níveis da hierarquia universitária. O trabalho de Bradley (2016) também aborda o quadro docente das universidades, identificando a falta de um alinhamento claro entre a estratégia organizacional e como o talento acadêmico é recrutado, desenvolvido, retido e recompensado. Para Sanusi, Irianto e Sumiyati (2019), há evidências de que as universidades devem considerar a implementação de fatores de liderança visionária para melhorar o desempenho da gestão de recursos humanos nas universidades.

A gestão estratégica é um processo contínuo e interativo que visa manter uma organização como um conjunto apropriadamente integrado a seu ambiente (Caggy \& Benevides, 2019). Professores de diferentes áreas e servidores administrativos possuem carreiras divergentes e merecem especial atenção para suas necessidades. Somado a isto, há a singularidade da gestão de instituições universitárias, devido ao seu alto grau de autonomia (Altbach, 1987).

Para Medne, Lapina e Zeps (2020), as universidades estão em busca de soluções para aumentar sua eficiência e melhorar a qualidade de seus serviços, considerando abordagens de desenvolvimento de sistemas de qualidade que se vinculem à estratégia. A abordagem que melhor se adapta à instituição deve estar alinhada com os objetivos estratégicos da instituição, cultura, política de qualidade e indicadores-chave de desempenho. Isto engloba também a gestão de recursos humanos, visto que são as pessoas que constroem a cultura das organizações e contribuem para atingir os indicadores de desempenho. Na seção seguinte, passa-se aos métodos utilizados neste estudo.

\section{Procedimentos Metodológicos}

Com o intuito de caracterizar o estado do conhecimento acerca da Gestão Estratégica de Pessoas no contexto das universidades, a natureza desta pesquisa é bibliográfica e descritiva, contempla, assim, uma abordagem qualitativa (Alyrio, 2009; Vergara, 2007).

Realizou-se um levantamento para constituir o corpus bibliográfico do estudo, no qual foram determinadas as bases de dados $\operatorname{Scopus}^{\circledR}$ e Web of Science ${ }^{\circledR}$ para a realização das buscas dos trabalhos. Estas bases foram selecionadas por contemplarem artigos provenientes de periódicos nacionais e internacionais, com reconhecida aderência aos estudos organizacionais, bem como com fator de impacto junto às áreas de Administração e de Educação.

Os artigos foram obtidos por meio dos os seguintes critérios: Primeiramente foi realizada uma busca por artigos completos, que contivessem as palavras "strategic management"; "human resource management" e "higher education", ambas combinadas pelo operador booleano "AND", nos últimos cinco anos completos. Ou seja, desde 2015 até 2020. Não foi definido um limite de abrangência de área. Para os idiomas dos trabalhos, foram 
selecionados os que estavam em português, inglês e espanhol e, para o tipo de trabalho, contemplou-se artigos completos revisados por pares. Este levantamento foi realizado nos dias 5, 6 e 13 de setembro de 2020.

Os artigos do corpus bibliográfico de ambas as bases foram tratados por meio dos seguintes critérios de exclusão por filtro, em que foi considerada a aderência necessária ao objetivo de estudo: a) por título; b) por resumo; c) por leitura na íntegra. Vale destacar que, houve a rejeição de duplicatas nas bases utilizadas e, para a leitura na íntegra, foram considerados os seguintes elementos-chave: a) objetivo geral; b) fundamentação teórica; c) métodos adotados; d) principais resultados; e) conclusões; f) proposta de estudos futuros.

Posteriormente, para analisar os trabalhos selecionados, utilizou-se da Análise de Conteúdo por categorização analítica (Bardin, 2011). Nesta foram identificadas as dimensões teóricas presentes na amostra. Ademais, foram descritas as contribuições dos estudos analisados com o tema de estudo, de maneira a identificar possíveis lacunas sobre o que ainda poderia ter sido pesquisado sobre o assunto.

Além disso, para fins de análise de dados da pesquisa em termos bibliométricos, utilizou-se da estatística descritiva, por meio de gráficos e tabelas (Dancey \& Reidy, 2013). Os dados da amostra foram, então, discutidos com base nos seguintes elementos-chave: a) índice de citações das obras; b) países de origem dos autores das obras; c) instituições de vínculo dos autores; d) métodos de pesquisa utilizados. Por fim, na seção seguinte, tais dados são discutidos.

\section{Apresentação de Dados e Resultados}

Nesta seção, traz-se os dados e as discussões relacionadas à gestão estratégica de pessoas nas universidades, sob a perspectiva do processo de conhecimento sobre o tema. Inicia-se pela apresentação de dados referentes ao quantitativo de artigos encontrados nas bases de dados Scopus ${ }^{\circledR}$ e Web of Science ${ }^{\circledR}$, conforme descrito nas Tabelas 1 e 2.

Tabela 1

Palavras-chave: strategic management and higher education

\begin{tabular}{lcccc}
\hline \multicolumn{1}{c}{ Base de dados } & Amostra & Filtro por título & Filtro por resumo & Filtro por leitura na íntegra \\
\hline Web Of Science & 85 & 45 & 28 & 5 \\
Scopus & 118 & 33 & 16 & 3 \\
\hline Total geral & 203 & 78 & 44 & 8 \\
\hline
\end{tabular}

Nota: Dados da pesquisa (2020).

Tabela 2

Palavras-chave: human resource management and higher education

\begin{tabular}{lcccc}
\hline \multicolumn{1}{c}{ Base de dados } & Amostra & Filtro por título & Filtro por resumo & Filtro por leitura na íntegra \\
\hline Web Of Science & 89 & 22 & 18 & 3 \\
Scopus & 149 & 35 & 10 & 2 \\
\hline Total geral & 238 & 57 & 28 & 5 \\
\hline
\end{tabular}

Nota: Dados da pesquisa (2020). 
De acordo com o que consta nas Tabelas 1 e 2, observa-se que dos 441 artigos levantados a partir das combinações de palavras-chave relacionadas ao objetivo de estudo e após os critérios de exclusão por título, resumo e por leitura na íntegra, restaram 13 artigos que apresentaram aderência à pesquisa. Diante disso, a seguir são apresentadas as análise qualitativas da amostra em termos de dimensões teóricas da gestão estratégica de pessoas, as quais foram contempladas pelos autores dos trabalhos selecionados, conforme a Figura 1.

Figura 1

Dimensões Teóricas da Gestão Estratégica de Pessoas nas Universidades

\begin{tabular}{llc}
\hline \multicolumn{1}{c}{ Dimensão teórica } & \multicolumn{1}{c}{ Agentes organizacionais } & Ferramentas/abordagens estratégicas \\
\hline Gestão da Mudança & $\begin{array}{l}\text { Professores, gestores e demais } \\
\text { funcionários }\end{array}$ & TQM e Análise SWOT \\
Desempenho Organizacional & $\begin{array}{c}\text { Gerentes, supervisores, gestores do topo } \\
\text { Indicadores de desempenho estratégico, } \\
\text { inovação como abordagem estratégica, } \\
\text { Gficácia Gerencial }\end{array}$ & gestão do conhecimento como ferramenta \\
Gestão de Talentos & Gestores do topo & estratégica \\
& Professores e pesquisadores & Liderança estratégica \\
Vantagem competitiva & O método de Eliminação e Escolha Expressando \\
\hline
\end{tabular}

Nota: Dados da Pesquisa (2020).

A gestão da mudança (Figura 1) emergiu na amostra vinculada a duas perspectivas (Doyle \& Brady, 2018; Sanusi et al., 2019). Na Primeira, ressalta-se que modelos de gestão adotados em instituições de ensino superior (IES) têm sido baseados em abordagens racionais quanto à gestão da mudança, a partir da importação de preceitos corporativos tradicionais (Doyle \& Brady, 2018).

No entanto, tal gestão nas IES pode ser tratada sob paradigmas alternativos, nos quais modelos organizacionais distintos tratam dos padrões de gestão de mudanças sob enfoques holísticos e de mudança contínua. A ênfase da gestão estratégica nesse tipo de instituição recai sobre políticas que contemplam a liderança, o que se estende para a gestão de pessoas. Envolve, portanto, todos os agentes de mudança (docentes, gestores, funcionários) "em conversas organizacionais, revelando diferenças e padrões e encorajando a experimentação com ideias emergentes" (Doyle \& Brady, 2018, p. 17).

Na segunda perspectiva, aborda-se a gestão da mudança nas IES em alinhamento com a gestão da qualidade, sob o prisma desta última como base para a gestão da governança universitária. Diante disso, ressalta-se a necessidade de as universidades investirem em processos de liderança vinculados à gestão de pessoas, com foco para os gestores, o que se caracteriza como estratégico no contexto de indicadores de qualidade, frente às pressões de seu ambiente de atuação (Sanusi et al., 2019). 
Abordagens e ferramentas estratégicas são incentivadas, tais como a Total Quality Management (TQM) e a Análise SWOT, respectivamente, entre outras. O intuito é fomentar ações de gestão de pessoas que levem ao empoderamento dos agentes organizacionais, o que perpassa pela qualidade da estrutura organizacional e de tecnologias. São, também, apontados os aspectos contributivos do papel da liderança nos processos de mudança. Ou seja, a gestão estratégica ocorre por meio de elementos de gestão e de liderança, adequados e relacionados ao potencial da educação superior, que pode ser maximizado para atingir seus objetivos (Sanusi et al., 2019).

A segunda dimensão identificada na amostra é a do desempenho organizacional. Ela aparece aplicada à gestão estratégica de pessoas nas universidades de forma relacional com a teoria do capital intelectual (Cl), o qual também teve destaque na dimensão da vantagem competitiva, descrita a seguir. São contemplados os ajustes de indicadores de $\mathrm{Cl}$, sob três aspectos: capital humano (stakeholders internos), capital estrutural (interno) e capital relacional; este voltado à relação com colaboradores (stakeholders externos) e ambientes externos às organizações (E. Pedro, Leitão, \& Alves, 2019; Secundo, Dumay, Schiuma, \& Passiante, 2016; Secundo, Elena-Perez, Martinaitis, \& Leitner, 2015; Tjahjadi, Soewarno, Astri, \& Hariyati, 2019; Zhao \& Tao, 2017).

A dimensão do desempenho também está associada com a satisfação no trabalho. Esta é considerada como um indicador de desempenho relevante para a gestão estratégica de pessoas, dado que implica o comprometimento organizacional (Veloso, Lunga, \& Fernandes, 2017). Cabe, então, aos gestores educacionais atentarem para essa abordagem da qualidade de vida nas organizações. Adaptar continuamente práticas organizacionais e de liderança gerencial conduz à busca por equilíbrio entre interesses que podem ser partilhados por colaboradores e organizações, o que reflete nas estratégias organizacionais (Veloso et al., 2017).

Outra abordagem da gestão de desempenho é a que dialoga com a gestão do conhecimento e da inovação, as quais foram consideradas como ferramentas estratégicas para a gestão de pessoas nas universidades (Govender, Perumal, \& Perumal, 2018). Ambas as perspectivas são tratadas de maneira processual. Volta-se esforços para a retenção de expertise e inteligência coletivas nas organizações, as quais são utilizadas para promover a inovação por meio da aprendizagem organizacional (Govender et al., 2018).

Com relação à eficácia gerencial, está relacionada ao poder que a liderança exerce para que as estratégias sejam bem conduzidas e a organização atinja seus objetivos. A atenção à liderança é característica da gestão de pessoas, e os autores Secundo et al. (2015) abordam este tema ao propor um modelo ligado ao capital humano e à eficácia gerencial. Tyagi e Moses (2020) trabalham bem esta dimensão teórica ao desenvolver uma escala com a finalidade de medir a eficácia gerencial de líderes de instituições de ensino na Índia. Nesta dimensão são abordados aspectos como a gestão de pessoas a partir da liderança gerencial, tarefas gerenciais e gestão de relacionamento. A eficácia gerencial depende de uma série de fatores, como o poder que o líder possui (remuneração, promoções e conhecimento, etc.), contatos pessoais (networking), relações interpessoais (boa comunicação) e comportamento (Tyagi \& Moses, 2020).

As instituições de ensino superior precisam não apenas contratar talentos, mas também gerenciar o ciclo de vida de seus talentos de forma estratégica (Al-Abbasi \& Masri, 2020). Na dimensão da gestão de talentos, Al- 
Abassi e Masri (2020) estudam o corpo docente de um college de negócios da Europa, e afirmam que deve haver uma avaliação do alinhamento, capacidades e envolvimento do corpo docente atual a fim de se obter um "corpo docente nota 10". Neste cenário, as IES devem desenvolver processos, políticas e comportamentos necessários para aprimorar, mudar e promover o desenvolvimento do corpo docente e a otimização de talentos (Al-Abbasi \& Masri, 2020).

Ainda sobre a gestão de talentos, Bradley (2016) critica a situação atual do quadro de professores de uma instituição na Austrália, por falta de um alinhamento claro entre a estratégia organizacional e como o talento acadêmico é recrutado, desenvolvido, retido e recompensado. Com isso, os autores demonstram a importância da gestão de pessoas no recrutamento e principalmente, na manutenção de seu quadro de talentos. Pois as pessoas possuem necessidades e obrigações que devem ser avaliadas e acompanhadas periodicamente, com uma boa gestão envolvida com a estratégia da organização.

As instituições que se preocupam com isso, pode-se afirmar que adquirem uma vantagem competitiva frente às demais. Sobre a abordagem da vantagem competitiva, nas IES, esta pode ser medida a partir da gestão dos componentes de capital intelectual, que pode potencializar por meio da valorização do conhecimento (Marulanda Grisales, Grajales, \& Correa, 2018). Tjahjadi et. al. (2019) enfatizam que o capital intelectual é a base da vantagem competitiva, demonstrando a relação dela com a gestão de pessoas e a estratégia das organizações que desejam se diferenciar positivamente.

Por meio das dimensões teórica da amostra ora apresentadas, encontrou-se que o tema da gestão estratégica de pessoas esteve vinculado a aspectos da gestão estratégica que consideram o papel gerencial e de liderança nas universidades. Isso ocorreu, no sentido de que cabe ao agente detentor das características do líder a promoção de elos entre a gestão de pessoas e os demais sistemas organizacionais.

Considerado, ainda, o capital humano no cumprimento de estratégias, o líder assume um papel de impulsionador de mudanças necessárias ao contexto dos desafios e pressões que as instituições universitárias enfrentam em seus ambientes de atuação. Dessa maneira, as lideranças nas universidades devem utilizar processos e ferramentas estratégicas que possibilitem as condições de desempenho voltadas à sua sustentabilidade, tanto para as instituições privadas como públicas, a partir das demandas da sociedade.

Como lacunas voltadas ao tema deste trabalho, a partir das contribuições dos autores da amostra, pode-se citar:

a. a necessidade de uma visão descentralizada sobre como a gestão estratégica de pessoas, a qual pode contemplar a participação de agentes sociais de outros níveis da organização, para além dos gestores. Ou seja, pode-se pensar em diálogos com aqueles que implementam estratégias, ampliando-se, ainda, o que foi encontrado sobre aspectos da satisfação de membros do corpo técnico e docente das universidades;

b. a falta de uma reflexão crítica sobre como o modelo hegemônico de capitalismo tem sido imposto dentro das universidades, implicando uma lógica de gestão de pessoas, com suas respectivas 
estratégias, voltada à mercantilização das relações de trabalho nas universidades, o que contribui para o aumento de problemas de saúde tanto para docentes como técnicos, sem contar o impacto na qualidade da prestação de serviços à comunidade universitária. Esse olhar alternativo vai ao encontro das abordagens holísticas para a estratégia organizacional, também comentadas na análise de dados.

Após verificar as dimensões teóricas abordadas, é interessante observar de que modo elas foram trabalhadas. Assim, no que se refere aos aspectos metodológicos das pesquisas analisadas, as dimensões estudadas foram abordadas de maneira qualitativa e quantitativa, pelos autores. A Figura 2 demonstra quantos artigos utilizaram cada uma das abordagens:

Figura 2

Abordagem de Pesquisa dos Artigos da Amostra

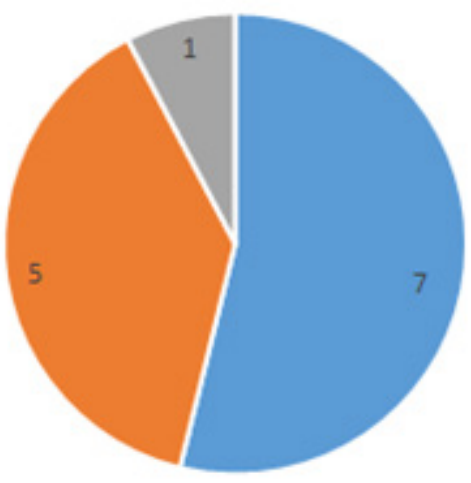

- Qualitziva = Quantitativa = QualitaivaQuantitativa

Nota: Elaborado pelos autores.

A pesquisa qualitativa é a mais utilizada quando se estuda gestão estratégica e gestão de pessoas, em periódicos de alto impacto, nos últimos 5 anos. Foram 7 (sete) artigos, enquanto na pesquisa quantitativa foram 5 (cinco) trabalhos. Um trabalho apenas utilizou os dois métodos, sendo uma pesquisa com abordagem quali-quanti (Veloso; Lunga; Fernandes, 2017).

O destaque da abordagem qualitativa nos trabalhos, vem ao encontro de uma característica observada por Ichikawa e Santos (2006), que afirmam que há uma valorização de estudos de cunho mais qualitativo em disciplinas das ciências humanas e sociais.

Quanto às técnicas de coleta e análise de dados, os métodos que mais chamam atenção na abordagem qualitativa são: Estudo de caso (Al-Abbasi \& Masri, 2020; Pedro et al., 2019; Tyagi \& Moses, 2020), e revisão teórica da literatura, discutindo e refletindo sobre o tema (Secundo et al., 2016; Veloso et al., 2017).

Na abordagem quantitativa há trabalhos que utilizam métodos de estatística mais avançados, como a Modelagem de equações estruturais (Tjahjadi et al., 2019) e outros mais difundidos, como a estatística descritiva (Zhao \& Tao, 2017) e escala Likert (Govender et al., 2018).

Foi observado também que um ponto bastante estudado nesta amostra, em relação às teorias voltadas à gestão estratégica de pessoas, foi o Capital Intelectual. Nos trabalhos é destacado que o capital intelectual é um conceito que vem ganhando bastante força, em razão da era do conhecimento em que vivemos. Assim, o capital 
humano, a inovação, o registro de patentes, ganham muita importância nas estratégias que as universidades definem para seu crescimento. O capital humano é o capital intelectual bastante citado pelos autores, e está alinhado à gestão de pessoas, como visto nos trabalhos de Secundo et. al (2015), Secundo et. al. (2016), Grisales et. al. (2018) e Pedro, Leitão e Alves (2019).

Ademais, em termos de análise bibliométrica da amostra, são descritos os seguintes elementos-chave: a) ano de publicação dos artigos da amostra - Figura 3; b) índice de citações das obras - Tabela 3; c) periódicos que publicaram sobre o tema - Tabela 4; d) países de origem dos autores das obras - Figura 4.

\section{Figura 3}

Ano de Publicação dos Artigos da Amostra

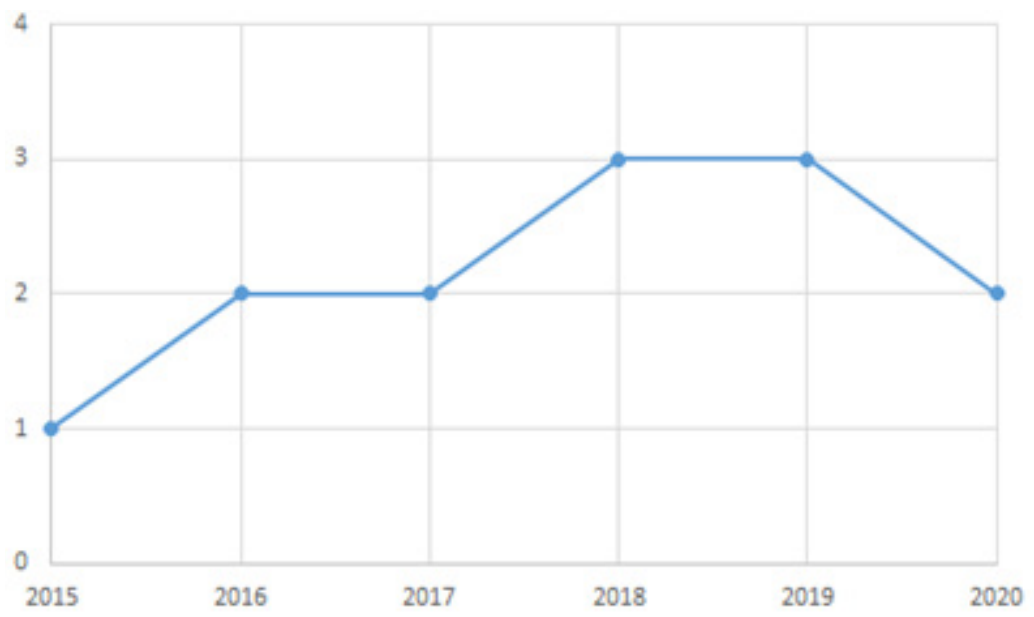

Nota: Elaborado pelos autores.

O Gráfico 1 apresenta os anos de publicação dos artigos da amostra. É demonstrado que o assunto gestão estratégica e gestão de pessoas está recebendo mais importância dos periódicos, visto o gráfico ser crescente. O ano de 2020 apresenta uma pequena queda em razão de ainda estar em curso, e assim novos artigos ainda podem ser publicados. Com o aumento do interesse pelo tema, por parte da academia, constata-se a relevância de pesquisas envolvendo este tema.

Ainda sobre a importância da pesquisa a respeito do tema, na Tabela 3 apresenta-se os artigos mais citados nos últimos cinco anos, e que compõem esta amostra:

\section{Tabela 3}

Índice de citações das obras

A dynamic approach (2015)

Managing intellectual capital through a collective intelligence approach An integrated framework for universities (2016)

Talent management for universities (2016) 
Artigo

Na de citações

Reframing the university as an emergent organisation: implications for strategic management and

leadership in higher education (2018)

The intellectual capital of higher education institutions Operationalizing measurement through a strategic

prospective lens (2019)

Does intellectual capital matter in performance management system-organizational performance

relationship? Experience of higher education institutions in Indonesia (2019)

Model of the empowerment of governance based on the human resource management for supply chains in higher education (2019)

Total

Nota: Elaborado pelos autores.

Conforme demonstrado na Tabela 3, na amostra há oito artigos com citações em outras obras. Destes oito, se destacam os artigos de Secundo et. al. (2015) e Secundo et. al. (2016) com 67 e 58 citações, respectivamente. O trabalho dos italianos também é mais antigo que os demais da amostra, o que pode justificar ter recebido maior número de citações, já que as outras pesquisas são mais recentes. Inclusive, ambos artigos foram publicados na revista britânica Journal of Intellectual Capital, que é o periódico que mais tem publicado sobre o tema, conforme demonstra a Tabela 4:

Tabela 4

Periódicos que Publicam sobre o Tema

Periódico

Journal of Intellectual Capital

AD-minister

Australian Universities' Review

International Journal of Leadership in Education

International Journal of Supply Chain Management

Journal of Discrete Mathematical Sciences and Cryptography

Journal of Higher Education Policy and Management

Management Decision

SA Journal of Information Management

TOJET: The Turkish Online Journal of Educational Technology

TOTAL

Nota: Elaborado pelos autores.

Enquanto o Journal of Intellectual Capital publicou quatro artigos da amostra, os demais artigos foram publicados em revistas variadas, com apenas uma publicação cada um. Este periódico tem fator de impacto JCR 5.330, que é bastante alto, o que valida a importância de pesquisas que envolvem o tema gestão estratégica de pessoas. As demais publicações são de diversos países, tais como África do Sul (SA Journal of Information Management) e Turquia (The Turkish Online Journal of Educational Technology), demonstrando haver interesse no tema por parte de editores de todo o mundo. 
A variedade de países na amostra também fica explícita quando analisamos o país de origem dos autores. A Figura 4 apresenta tais informações.

Figura 4

Países de Origem dos Autores das Obras

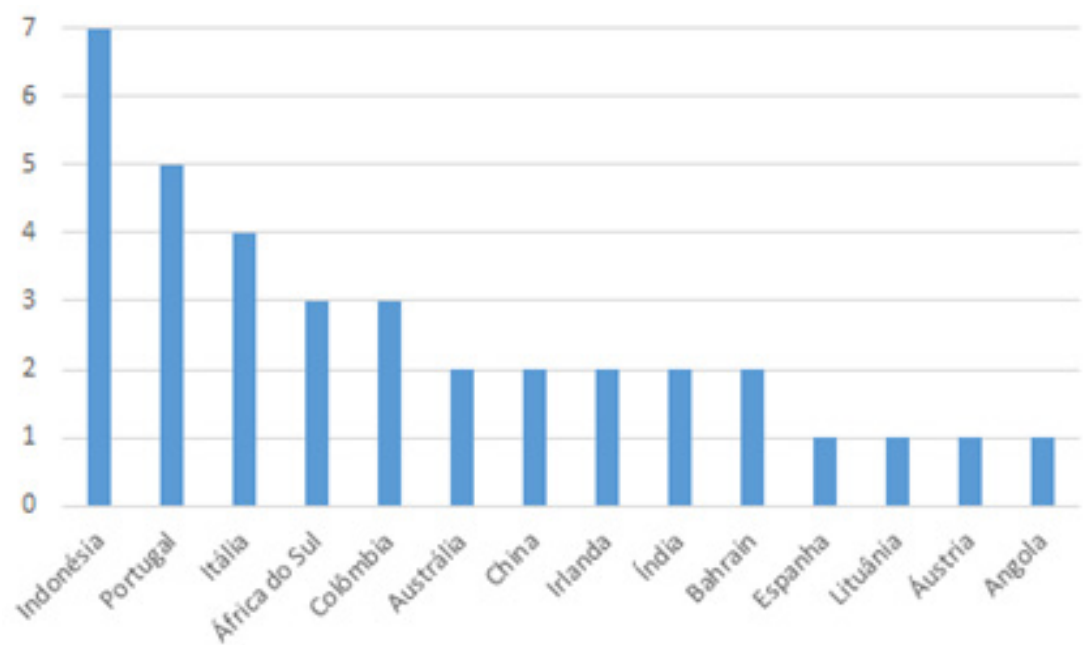

Nota: Elaborado pelos autores.

A Figura 4 apresenta 14 nacionalidades de autores para os 13 artigos da amostra. Isto porque os artigos da amostra foram em sua maioria o resultado de pesquisa elaborada por mais de um autor. A Indonésia se destaca com sete autores, pois há dois artigos oriundos deste país, um com quatro (Tjahjadi et al., 2019) e outro com três autores (Sanusi et al., 2019). Este perfil de haver mais de um pesquisador no artigo, porém todos da mesma nacionalidade, é o que acontece na maioria dos artigos da amostra.

Entretanto, é interessante destacar quando aparecem as redes de pesquisa. Isto foi observado no caso da Itália, que com dois artigos (Secundo et al., 2016, 2015), apresentou parcerias com pesquisadores da Lituânia, Espanha e Áustria. O mesmo com os pesquisadores de Portugal, que pesquisaram juntamente com Angola (Veloso et al., 2017). A seguir, passa-se às considerações finais deste trabalho.

\section{Considerações Finais}

Este estudo se propôs a caracterizar o processo do conhecimento sobre a gestão estratégica de pessoas no contexto das universidades. Para tanto, tratou-se da produção acadêmica in sobre o tema, com enfoque em trabalhos publicados nas bases de dados Scopus ${ }^{\circledR}$ e Web of Science ${ }^{\circledR}$, no período de 2015 a 2020.

Destaca-se a quantidade de artigos científicos que abordam gestão estratégica, bem como gestão de pessoas nas universidades, porém ao conciliar estes dois temas, são poucos os trabalhos encontrados. Nesta pesquisa, a amostra final resultou em 13 artigos. Esta é uma lacuna de estudo encontrada, visto que a gestão de pessoas é um ponto estratégico para as universidades trabalharem, no intuito de melhorarem seu desempenho frente as demandas de seu ambiente de atuação.

Ademais, constatou-se interesse no tema, demonstrado pelo crescimento das pesquisas no decorrer dos anos, conforme apontado no Gráfico 1. Além disso, a variedade de países que pesquisaram sobre o tema demonstra uma valorização da estratégia na gestão de pessoas por todo o mundo, afirmando ser este um tema 
bastante atual para a academia internacional. Soma-se a isto, o periódico que mais publicou trabalhos envolvendo estratégia na gestão de pessoas em universidades, ser um journal com alto índice de impacto, concedendo credibilidade às pesquisas e o interesse acerca do assunto.

Os estudos da amostra destacam, também, a liderança e a satisfação no trabalho como estratégias de motivação e melhora no desempenho da gestão de pessoas nas universidades. Isto se justifica pelo fato de que cabe ao agente detentor das características do líder a promoção de elos entre a gestão de pessoas e os demais sistemas organizacionais que compõem o plano estratégico das universidades.

O capital intelectual também é bastante discutido nos trabalhos relacionados à estratégia; enfatiza-se a importância das pessoas para o sucesso das organizações, no caso, da gestão das universidades. Não basta apenas focar em como melhorar processos, rotinas, se não pensar nas pessoas que executarão essas ferramentas. $\mathrm{O}$ capital intelectual é assim, matéria-prima essencial para organizações da era do conhecimento.

Diante do fato de que não foi encontrada nenhuma revisão sistemática sobre a temática abordada, esta pesquisa auxiliou no preenchimento desta lacuna do conhecimento. Os processos de busca foram delimitados anteriormente à condução da revisão, permitindo assim a obtenção de resultados imparciais e passíveis de reprodução.

Dentre as lacunas de pesquisa identificadas a partir do levantamento sistemático da teoria destacam-se: a falta de pesquisas brasileiras que contemplem a realidade das universidades brasileiras, estudando a estratégia voltada à gestão de pessoas em universidades públicas, que trabalhem a motivação e a satisfação no trabalho, bem como a liderança e o desempenho em servidores que são concursados.

Há também um maior número de pesquisas que se atentam aos funcionários docentes apenas, focando na carreira e na manutenção destes talentos que são estratégicos para a universidade. Porém, cabe destacar que são necessários estudos que contemplem também os agentes administrativos, que muitas vezes são os responsáveis por executar as estratégias pensadas na gestão universitária. Além disso, é preciso aprofundar a crítica de como a lógica capitalista vem ditando os rumos dos sistemas de gestão organizacional, algo que também tem sido pouco discutido sobre a temática. Estas lacunas são também recomendações para estudos futuros envolvendo a temática, no sentido de pensarem o campo da gestão universitária sob o prisma da gestão de pessoas como um sistema estratégico para o alcance de objetivos. 


\section{Referências}

Al-Abbasi, S., \& Masri, H. (2020). Optimizing faculty talents through identifying entrepreneurial champions: an ELECTRE IV approach. Management Decision. https://doi.org/10.1108/MD-09-2019-1305

Altbach, P. G. (1987). The knowledge context: comparative perspectives on the distribution of knowledge. Albany: State University of New York Press.

Alyrio, D. R. (2009). Métodos e técnicas de pesquisa em administração. Rio de Janeiro: Fundação CECIERJ.

Andersen, T. J. (2013). Short introduction to strategic management. Cambridge: Cambridge University Press.

Ansoff, H. I. (1965). Corporate strategy: an analytic approach to business policy for growth and expansion. New York: McGraw-Hill Companies.

Balogun, J., Jacobs, C., Jarzabkowski, P., Mantere, S., \& Vaara, E. (2014). Placing Strategy Discourse in Context: Sociomateriality, Sensemaking, and Power. Journal of Management Studies, 51(2), 175-201. https://doi. org/10.1111/joms.12059

Bardin, L. (2011). Análise de conteúdo. São Paulo: Edições 70.

Barnard, C. I. (1938). The functions of the executive. Cambridge, MA: Harvard Universty Printing Office.

Boas, A. A., \& Andrade, R. (2009). Gestão estratégica de pessoas. Rio de Janeiro: Elsevier.

Bontis, N. (1996). There's a price on your head: Managing intellectual capital strategically. Yves Business Quarterly, 60(94), 41-47.

Bradley, A. (2016). Talent management for universities. Australian Universities' Review, 58(1), 13-19.

Caggy, R. C., \& Benevides, T. M. (2019). Strategic canvas: conduza a estratégia do seu negócio por caminhos dinâmicos e criativos de forma inovadora. Rio de Janeiro: Alta Books Editora.

Chandler, A. D. (1962). Strategy and structure: chapters in the history of the industrial enterprise. MIT Press, 120, 519-551. https://doi.org/10.2307/3111403

Fonseca, D. R., Meneses, P. P. M., Filho, A. I. da S., \& Campos, N. G. (2013). Autonomia para gestão estratégica de pessoas no setor público federal: Perspectivas de análise e agenda de pesquisa. Revista de Administracao Publica, 47(6), 1451-1475. https://doi.org/10.1590/S0034-76122013000600006

Daley, D. M. (2016). Strategic Human Resource Management. In N. M. Riccucci (Ed.), Public Personnel Management: Current concerns, Future challenges (p. 120). New York: Routledge Taylor \& Francis Group.

Dancey, C. P., \& Reidy, J. (2013). Estatística sem matemática para psicologia. Porto Alegre: Penso.

Doyle, T., \& Brady, M. (2018). Reframing the university as an emergent organisation: implications for strategic management and leadership in higher education. Journal of Higher Education Policy and Management, 40(4), 305-320. https://doi.org/10.1080/1360080X.2018.147860

Drucker, P. (1954). Management. Business Horizons. New York: Harper and Row.

Dutra, A. (2014). Gestão estratégica de pessoas. Palhoça: Unisul Virtual.

Easterby-Smith, M. (1987). Change and innovation in higher education: a role for corporate strategy? Higher Education, 16(1), 37-52. https://doi.org/10.1007/BF00139246 
Elbanna, S. (2006). Strategic decision making : process perspectives. International Journal of Management Reviews, 8(1), 1-20. https://doi.org/10.1111/j.1468-2370.2006.00118.x

Etzioni, A. (1972). Organizações modernas. (M. L. M. Leite, Trad.) (2nd ed.). São Paulo: Pioneira.

Farjoun, M. (2002). Towards an organic perspective on strategy. Strategic Management Journal, 23, 561-594. https://doi.org/10.1002/smi.239

Golsorkhi, D., Rouleau, L., Seidl, D., \& Vaara, E. (2015). Cambridge Handbook of Strategy as Practice. Organization Studies (Vol. 32).

Govender, L. N., Perumal, R., \& Perumal, S. (2018). Knowledge management as a strategic tool for human resource management at higher education institutions. SA Journal of Information Management, 20(1), 1-10. https:// doi.org/10.4102/sajim.v20i1.966

Hamel, G., \& Prahalad, C. K. (1994). Competing for the future. Harvard Business Review, (jul-ago), $122-128$.

Hasani, K., \& Sheikhesmaeili, S. (2016). Knowledge management and employee empowerment. Kybernetes, 45(2), 337-355.

Herrero Filho, E. (2017). Balanced scorecard e a gestão estratégica: uma abordagem prática. Rio de Janeiro: Alta Books Editora.

Huff, A. S., \& Reger, R. K. (1987). A review of strategy process research. Journal of Management, 13(2), $211-236$. Ichikawa, E. Y., \& Santos, L. W. (2006). Pesquisa Qualitativa em Estudos Organizacionais. In C. K. Godoi, R. Bandeira de Mello, \& A. B. Silva (Eds.), Pesquisa qualitativa em estudos organizacionais (pp. 181-205). São Paulo: Saraiva.

Jarzabkowski, P. (2005). Strategy as practice. London: Sage Publications.

Jarzabkowski, P., \& Kaplan, S. (2015). Strategy tools-in-use: A framework for understanding "technologies of rationality" in practice. Strategic Management Journal, 36(4), 537-558. https://doi.org/10.1002/smj.2270

Juul Andersen, T., \& Minbaeva, D. (2013). The role of human resource management in strategy making. Human Resource Management, 52(5). https://doi.org/10.1002/hrm.21562

Keller, G. (1983). Academic strategy. Baltimore: Johns Hopkins.

Kotler, P., Berger, R., \& Bickhoff, N. (2010). The quintessence of strategic management: What you really need to know to survive in business. The Quintessence of Strategic Management: What You Really Need to Know to Survive in Business (Vol. 1993). Heidelberg: Springer. https://doi.org/10.1007/978-3-642-14544-5

Lei $n^{\circ}$ 10.861, de 14 de Abril de (2004). Institui o Sistema Nacional de Avaliação da Educação Superior - SINAES e dá outras providências. Recuperado de http://www.planalto.gov.br/ccivil 03/ ato2004-2006/2004/lei/l10.861. htm em: 22 set. 2020.

Marulanda Grisales, N., Grajales, C. L. R., \& Correa, J. E. (2018). Management of intangible assets of relational capital in higher educations institutions. AD-Minister, 5(33), 85-112. https://doi.org/10.17230/ad-minister.33.5

Massen, P. A. M., \& Potman, H. P. (1990). Strategic decision making in higher education an analysis of the new planning system in dutch higher education. Higher Education, 20, 393-410. 
Medne, A., Lapina, I., \& Zeps, A. (2020). Sustainability of a university's quality system: adaptation of the EFQM excellence model. International Journal of Quality and Service Sciences, 12(1), 29-43. https://doi. org/10.1108/IJOSS-09-2019-0108

Mintzberg, H., Ahlstrand, B., \& Lampel, J. (1998). Strategy safari: a guided tour through the wilds of strategic management. New York: Bookman. https://doi.org/0684847434

Mintzberg, H., \& Lampel, J. (1999). Reflecting on the strategy process. Sloan Management Review, 40(3), 21-30. https://doi.org/10.1128/JVI.78.19.10303-10309.2004

Mintzberg, H., \& Quinn, J. B. (2001). O processo da estratégia (3rd ed.). Porto Alegre: Bookman.

Mintzberg, H., \& Waters, J. A. (1985). Of strategies, deliberate and emergent. Strategic Management Journal, 6(3), 257-272. https://doi.org/10.1002/smj.4250060306

Pantoja, M. J., Camões, M. R. S., \& Bergue, S. T. (2010). Gestão de Pessoas: bases teóricas e experiências no setor público. Brasília: ENAP.

Pedro, E., Leitão, J., \& Alves, H. (2019). The intellectual capital of higher education institutions: Operationalizing measurement through a strategic prospective lens. Journal of Intellectual Capital, 20(3), 355-381. https:// doi.org/10.1108/JIC-07-2018-0117

Pedro, W. J. A. (2005). Gestão de Pessoas nas Organizações. Revista Brasileira Multidisciplinar, 9(2), 81. https://doi. org/10.25061/2527-2675/rebram/2006.v9i2.268

Peris-Ortiz, M., García-Hurtado, D., \& Devece, C. (2019). Influence of the balanced scorecard on the science and innovation performance of Latin American universities. Knowledge Management Research and Practice, 17(4), 373-383. https://doi.org/10.1080/14778238.2019.1569488

Pettigrew, A. M. (1992). The character and significance of strategy process research. Strategic Management Journal, 13(2 S), 5-16. https://doi.org/10.1002/smj.4250130903

Porter, M. E. (1980). Competitive strategy: techniques for analyzing industries and competitors. Competitive Strategy (Vol. 1). New York: The Free Press.

Qehaja, A. B., Kutllovci, E., \& Pula, J. S. (2017). Strategic management tools and techniques usage: a qualitative review. Acta Universitatis Agriculturae et Silviculturae Mendelianae Brunensis, 65(2), 585-600. https://doi. org/10.11118/actaun201765020585

Quinn, J. (1978). Strategic change: “logical incrementalism.” Sloan Management Review, 20(1), 7-21.

Robinson Jr., R. B., \& Pearce II, J. a. (1988). Planned patterns of strategic behavior and their relationship to businessunit performance. Strategic Management Journal, 9(1), 43. https://doi.org/10.1002/smj.4250090105

Rodrigues, L. M. A. (2017). Processo de formação e implementação das estratégias de um programa de pósgraduação em administração de uma universidade federal para atender ao sistema de avaliação da pósgraduação a partir da teoria das capacidades dinâmicas. Dissertação de Mestrado. Universidade Federal de Santa Catarina (UFSC), Florianópolis SC. 
Rodrigues, L. M. A., Moreira, K. D., \& Martins, C. B. (2020). Estratégias organizacionais no contexto da avaliação da pós-graduação brasileira. Estudos Em Avaliação Educacional, 31(77), 1-31.

Roesch, M. S. A. (2009). Projetos de estágio e de pesquisa em administração: guia para estágios, trabalhos de conclusão, dissertações e estudos de caso. São Paulo: Atlas S.A.

Sanusi, A., Irianto, S. Y., \& Sumiyati, L. (2019). Model of the empowerment of governance based on the human resource management for supply chains in higher education. International Journal of Supply Chain Management, 8(6), 671-680.

Secundo, G., Dumay, J., Schiuma, G., \& Passiante, G. (2016). Managing intellectual capital through a collective intelligence approach: An integrated framework for universities. Journal of Intellectual Capital, 17(2), 298319. https://doi.org/10.1108/JIC-05-2015-0046

Secundo, G., Elena-Perez, S., Martinaitis, Ž., \& Leitner, K. H. (2015). An intellectual capital maturity model (ICMM) to improve strategic management in European universities: A dynamic approach. Journal of Intellectual Capital, 16(2), 419-442. https://doi.org/10.1108/JIC-06-2014-0072

Souza, I. M. (2009). Gestão das universidades federais brasileiras: uma abordagem fundamentada na gestão do conhecimento. Tese de Doutorado. Florianópolis: Universidade Federal de Santa Catarina.

Tjahjadi, B., Soewarno, N., Astri, E., \& Hariyati, H. (2019). Does intellectual capital matter in performance management system-organizational performance relationship? Experience of higher education institutions in Indonesia. Journal of Intellectual Capital, 20(4), 533-554. https://doi.org/10.1108/JIC-12-2018-0209

Tyagi, N., \& Moses, D. B. (2020). Developing and validating the antecedents of managerial effectiveness: factorial constitution in institutions of higher learning. International Journal of Leadership in Education, 00(00), 1-21. https://doi.org/10.1080/13603124.2020.1762003

Veloso, C. M., Lunga, D. A., \& Fernandes, P. O. (2017). The Teachers' Satisfaction in Higher Education Institutions as Key Factor of the Strategic Management and of the Organizational Competitiveness. TOJET: The Turkish Online Journal of Educational Technology, December(Special Issue for INTE 2017).

Vergara, S. C. (2007). Projetos e relatórios de pesquisa em administração. São Paulo: Atlas S.A.

Walker, R. M. (2013). Strategic management and performance in public organizations: Findings from the miles and snow framework. Public Administration Review, 73(5), 675-685. https://doi.org/10.1111/puar.12073

Zhao, Y. Le, \& Tao, Z. (2017). Innovation strategy of human resources in colleges and universities in postmassification stage: evidence from Anhui province of China. Journal of Discrete Mathematical Sciences and Cryptography, 20(1), 377-388. https://doi.org/10.1080/09720529.2016.1183313 